\title{
FRONT PROPAGATION IN EVANESCENT MEDIA
}

\author{
M. Büttiker ${ }^{\mathrm{a}}$ and H. Thomas ${ }^{\mathrm{b}}$ \\ ${ }^{a}$ Département de Physique Théorique, Université de Genève, CH-1211 Genève 4, Switzerland \\ ${ }^{b}$ Institut für Physik, Universität Basel, CH-4056 Basel, Switzerland
}

\begin{abstract}
We investigate the time evolution of waves in evanescent media generated by a source within this medium and observed at some distance away from the location of the source. The aim is to find a velocity which describes a causal process and is thus, for a medium with relativistic dispersion, limited by the velocity of light. The wave function consists of a broad frequency forerunner generated by the onset of the source, and of a monochromatic front which carries the oscillation frequency of the source. For a medium with Schrödinger-like dispersion the monochromatic front propagates with a velocity which is in agreement with the traversal time, and in the relativistic case the velocity of the fronts is limited by the velocity of light. For sources with a sharp onset, the forerunners are not attenuated and in magnitude far exceed the monochromatic front. In contrast, for sources which are frequencyband limited, the forerunners are also attenuated and become comparable to the monochromatic front: like in the propagating case, there exists a time at which a broad frequency forerunner is augmented by a monochromatic wave.
\end{abstract}

PACS numbers: 05.45.+b, 72.10.Bg, 72.30.+q 
Fifteen years ago Rolf Landauer in collaboration with one of the authors [1] revived an old question: What is the time of interaction of a tunneling particle with the barrier? This work was motivated by the insight that the most often used approach which follows peaks of wave packets as they approach and emerge from a tunneling barrier is dubious: there is no causal relationship between the peak of an incident wave packet and the peak of an emerging wave packet. Instead a novel approach was needed: Ref. 1 and subsequent work [2,:3] investigated tunneling through a barrier with an oscillating potential and analyzed tunneling as a function of the oscillation frequency. Similarly, the Larmor clock, originally proposed by Baz [4] and Rybachenko [5], based on the precession of the spin of particles was re-analyzed and the importance of spin rotation was pointed out [6]. Both the oscillating barrier and the Larmor clock lead to traversal times which differ from a stationary phase analysis. We can indicate here the wide interest and the broad discussion which these works have generated only with a reference to a few recent works [7] and by a number of reviews 8 [11].

While there have been only a few experiments on electrical systems [12], recently a number of interesting experiments which exploit the analogy [13 between electron tunneling and tunneling of photons [14] or the propagation of classical microwaves in evanescent media [15 19] have provided a new impetus to the field. Of particular importance are the apparently superluminal tunneling times reported in these experiments. The superluminal appearance of wave packet peaks can be explained according to Deutch and Low [20] by demonstrating that the transmitted wave packet is made up mainly of contributions which stem from the head of the incident wave packet. Japha and Kurizki 21] explain that the connection between the transmitted wave packet and the leading portion of the incident wave is a consequence of destructive interference of causal retarded tunneling paths. It is clear that the wave evolution is inherently a causal process and that superluminal velocities arise from the use of an acausal definition of the velocity like the comparison of the peaks of the incident and the transmitted wave. These experiments thus raise the question of whether it is possible to define a traversal time which represents a manifestly causal process. We can identify a number of different velocities which characterize wave propagation like the phase velocity, the group velocity, the signal velocity and the velocity of the head of the wave. It is well understood that both the phase velocity and the group velocity can exceed the velocity of light, but that the signal velocity is always smaller than or at most equal to the velocity of light and the velocity of the head of the wave always coincides with the velocity of light. In principle there should exist a traversal time corresponding to each of these possible definitions of velocity. It is the purpose of this work to discuss a traversal time which in an evanescent medium corresponds to a signal velocity, and which, therefore, characterizes a causal process.

Interest in the signal velocity of propagating waves arose from the observation by $\mathrm{R}$. Wien that, in apparent contradiction to special relativity theory, the phase velocity and the group velocity can easily exceed the velocity of light in dispersive media. In response A. Sommerfeld 22] started a fundamental discussion by analyzing the propagation of wave fronts. While the phase velocity and the group velocity can exceed the velocity of light without contradicting the principles of causality and relativity, the velocity of the front and the signal velocity remain strictly smaller than or equal to the velocity of light [23]. A detailed discussion of this approach was subsequently given by Brillouin [24]. The method of Sommerfeld and 
Brillouin considers a source which is quiescent up to a given instant, when it is abruptly switched on. In a medium that allows propagation, the sudden onset of the source leads at the observation point located some distance away from the source to small forerunners of indefinite frequency; it is followed by a wave which oscillates with the frequency of the source and marks the arrival of the signal. For freely propagating waves (classically allowed regions of dispersion) the forerunners are small and the arrival of the main part is marked by a rapid increase in the intensity of the wave. The method of Sommerfeld and Brillouin was applied to evanescent media with a Schrödinger-like dispersion by Stevens [25]. Stevens calculates an arrival time of a signal. He did, however, not analyze the magnitude of the different contributions to the total wave. Indeed, subsequent works by Teranishi et al. [26], Jauho and Jonson [27], Ranfagni et al. 28] have called into question the very existence of a front which would mark the arrival of the signal in an evanescent medium. Moretti [29] emphasized exact analytical solutions, but similarly to Stevens did not discuss the magnitude of different contributions to the evolution of the wave. The lack of a main part found from numerical analysis [27,28] was further emphasized in more recent analytical work by Brouard and Muga 30]. In contrast to Stevens who predicted an arrival time of the main part of the wave with a traversal time which is in agreement with that found from the oscillating barrier or the Larmor clock, Ranfagni et al [28] and Brouard and Muga 30] suggest that their analysis is actually in better agreement with the phase time. Since propagation occurs now in an evanescent medium, it is essential to investigate the amplitudes of the front and the forerunners in detail. Contrary to the case of freely propagating waves, the evanescent medium transmits the high-frequency components which make up the forerunners with little attenuation (the forerunners propagate in effect freely) while the "main part" of the wave is evanescent and thus exponentially suppressed.

The situation changes dramatically if instead of a source with a sharp onset a frequencyband limited source is used. The source which is of interest switches on gradually but still fast compared to the traversal time. For a source which switches on too slowly, the traversal time cannot be resolved. Moreover, the highest frequency must be smaller than the threshold which permits free propagation. Thus the frequency-band is limited both at the high end and at the low end. For such a frequency-band limited source, all frequencies are in the evanescent range of the dispersion. This has the consequence that not only the fronts which carry the monochromatic frequency of the source, but also the forerunners are exponentially attenuated! The forerunners may, however, still exceed in magnitude the monochromatic fronts since the evanescent waves of the highest frequencies of the source are attenuated less strongly then the evanescent wave with the frequency of the source. Within this limitation, we encounter for the frequency-band limited source a wave evolution which is completely analogous to the freely propagating case: at the traversal time known from the oscillating barrier [1] and the Larmor clock [6], a forerunner of indefinite frequency is augmented by a nearly monochromatic wave of comparable amplitude. The discussion for the frequencyband limited source is presented for the case of a particle with Schrödinger-like dispersion. The discussion for a relativistic dispersion will be presented elsewhere [31]. 


\section{Source with a sharp onset}

We want to investigate propagation of a particle field $\psi(x, t)$ into a region $x>0$ with a constant potential $V$. The field equation-Schrödinger equation for a non-relativistic particle, Klein-Gordon equation for a relativistic boson, Dirac equation for a relativistic fermion-has plane-wave solutions

$$
\psi_{\omega}(x, t)=e^{-i[\omega t-i k(\omega) x]} \quad(\omega \text { real }),
$$

where the wave number $k(\omega)$ is determined by the dispersion relation

$$
\begin{gathered}
\hbar \omega=V+\frac{\hbar^{2} k^{2}}{2 m} \quad(\text { non-relativistic particle }) \\
(\hbar \omega-V)^{2}=\left(m c^{2}\right)^{2}+c^{2} \hbar^{2} k^{2} \quad \text { (relativistic particle). }
\end{gathered}
$$

$k(\omega)$ is real for propagating waves and imaginary for evanescent waves. Its sign is determined by the boundary condition that $\psi_{\omega}(x, t)$ is an outgoing wave in the $+x$-direction: Positive group velocity for propagating waves and exponential decay with $+x$ for evanescent waves yields

$$
\begin{aligned}
k(\omega) \operatorname{sign}(\omega)>0 & \text { for propagating waves } \\
\operatorname{Im} k(\omega)>0 & \text { for evanescent waves }
\end{aligned}
$$

For given $\omega$, the dispersion relations (2,3) have only a single root satisfying the boundary condition Eq. (4, 5).

Following Sommerfeld and Brillouin [23,24] and Stevens [25] we consider an arrangement which permits the investigation of wave front propagation: For $t<0$, the field is everywhere zero,

$$
\psi(x, t)=0 \quad(t<0) .
$$

At $t=0$, a source located at $x=0$ of frequency $\omega_{0}$ and amplitude $A$ is switched on, i.e.,

$$
\psi(0, t)=A(t) e^{-i \omega_{0} t} \quad \text { with } \quad A(t)=A \Theta(t)
$$

where $\Theta(t)$ is the step function, or in $\omega$-space,

$$
\hat{\psi}(0, \omega)=\hat{A}\left(\omega-\omega_{0}\right)=\int_{0}^{\infty} A(t) e^{i\left(\omega-\omega_{0}\right) t} d t=\frac{i A}{\omega-\omega_{0}+i 0^{+}} .
$$

The solution satisfying these initial conditions is

$$
\hat{\psi}(x, \omega)=\hat{A}\left(\omega-\omega_{0}\right) e^{i k(\omega) x} \quad(x>0),
$$

or in $t$-space,

$$
\psi(x, t)=\frac{1}{2 \pi} \int_{-\infty}^{+\infty} \hat{A}\left(\omega-\omega_{0}\right) e^{-i[\omega t-k(\omega) x]} d \omega .
$$


For the following, it is convenient to introduce a frequency $\Omega$ corresponding to the kinetic energy (plus rest energy in the relativistic case) of the particle,

$$
\hbar \Omega=\hbar \omega-V .
$$

Then, the solution may be written in the form

$$
\psi(x, t)=\frac{i A}{2 \pi} e^{-\frac{i}{\hbar} V t} \int_{-\infty}^{+\infty} \frac{1}{\Omega-\Omega_{0}+i 0^{+}} e^{-i \phi(\Omega ; x, t)} d \Omega
$$

where the phase function $\phi(\Omega ; x, t)$ is given by

$$
\phi(\Omega ; x, t)=\Omega t-k(\Omega) x .
$$

The integration is along the real $\Omega$-axis above the pole at $\Omega=\Omega_{0}-0^{+}$and above the branch cuts of the phase function $\phi(\Omega ; x, t)$. The strategy consists in shifting the path of integration into regions of the complex $\Omega$-plane where $\operatorname{Im} \phi(\Omega ; x, t) \rightarrow-\infty$ as $|\Omega| \rightarrow \infty$ and collecting any contributions from the pole and the branch cuts.

\section{Non-relativistic particle}

The phase function $\phi(\Omega)$ in the complex $\Omega$-plane is shown in Fig. 1. It has a branch cut $\Omega=(0 \ldots \infty)$. The values of the function $\phi(\Omega)$ on the lower sheet are related to those on the upper sheet by

$$
\phi^{\text {lower }}(\Omega)=\left[\phi^{\text {upper }}\left(\Omega^{*}\right)\right]^{*}
$$

The phase factor $e^{-i \phi(\Omega)}$ has a saddle point on the real axis. The saddle-point condition $d \phi /\left.d \Omega\right|_{s}=0$ yields

$$
\hbar \Omega_{s}=\frac{m}{2} \frac{x^{2}}{t^{2}}, \quad \hbar k_{s}=m \frac{x}{t}, \quad \hbar \phi_{s}=-\hbar \Omega_{s} t=-\frac{m}{2} \frac{x^{2}}{t}
$$

and the second derivative of the phase function at the saddle point is

$$
\phi_{s}^{\prime \prime}=\left.\frac{d^{2} \phi}{d \Omega^{2}}\right|_{s}=\frac{t}{2 \Omega_{s}}=\frac{\hbar}{m} \frac{t^{3}}{x^{2}} .
$$

The line stph of stationary phase $\operatorname{Re} \phi(\Omega)=\phi_{s}$, on which $\operatorname{Im} \phi$ decreases to $-\infty$ as one moves away from the saddle point, is the parabola

$$
\frac{\Omega_{i}}{\Omega_{s}}=\frac{1}{2}\left[1-\left(\frac{\Omega_{r}}{\Omega_{s}}\right)^{2}\right],
$$

where we have introduced the notation $\operatorname{Re} \Omega=\Omega_{r}, \operatorname{Im} \Omega=\Omega_{i}$. On the stph-line, the phase function is given by 


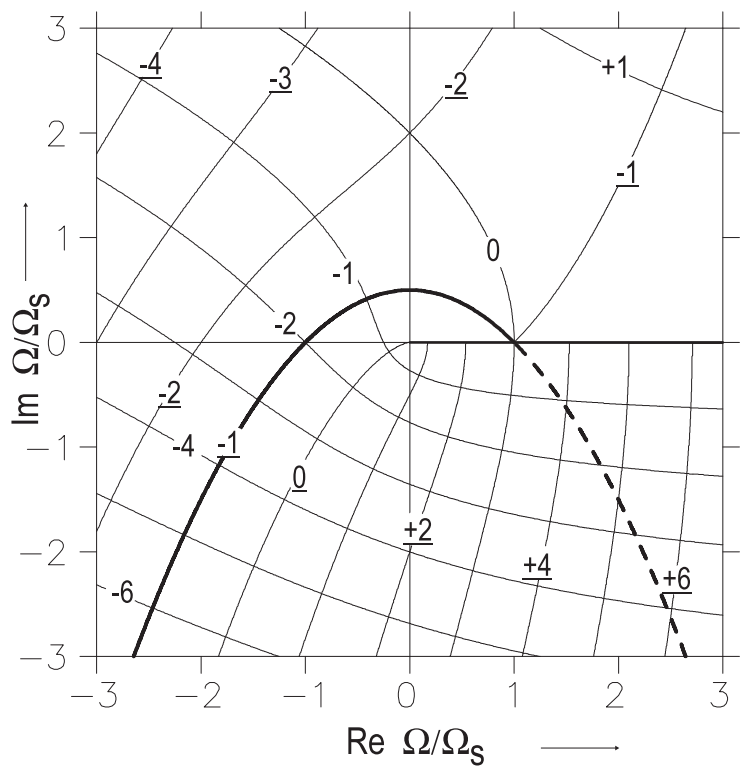

FIG. 1. Form of the phase function $\phi(\Omega)$ in the complex $\Omega$-plane for a non-relativistic particle. Shown are the lines of constant $\varphi_{r}=\operatorname{Re} \phi / \phi_{s}$ for $\varphi_{r}=-4 \ldots+6$ (underlined numbers) and $\varphi_{i}=\operatorname{Im} \phi / \phi_{s}$ for $\varphi_{i}=-6 \ldots+1$. The branch cut is indicated by an increased line thickness. The part of the line of stationary phase $\operatorname{Re} \phi=\phi_{s}$ on the upper and lower sheet is shown as a full and a dashed thick line, respectively. After Ref. [31].

$$
\phi(\Omega)=\phi_{s}\left[1+\frac{i}{2}\left(1-\frac{\Omega_{r}}{\Omega_{s}}\right)^{2}\right] .
$$

For a fixed point $x$ in space, the crossing points $\Omega= \pm \Omega_{s}$ of the $s t p h$-line with the real axis move inwards with increasing $t$. One of them crosses the pole $\Omega=\Omega_{0}$ at time $t=x / v_{m}$ where $v_{m}$ is a velocity given by 25]

$$
v_{m}=\sqrt{\frac{2}{m} \hbar\left|\Omega_{0}\right|} .
$$

We now deform the path of integration away from the real $\Omega$-axis such that it coincides with the stph-line. As long as $t<x / v_{m}$ there is no obstacle, and the integral remains unchanged; but for $t>x / v_{m}$ there occurs a loop around the pole which gives rise to a contribution $\psi_{p}$ to the wave function of the form

$$
\psi_{p}(x, t)=A e^{-i\left(\frac{1}{\hbar} V+\Omega_{0}\right) t} e^{i k\left(\Omega_{0}\right) x} \Theta\left(v_{m} t-x\right) .
$$

The integral along the stph-line yields a contribution $\psi_{s}$ to the wave function,

$$
\psi_{s}(x, t)=\frac{i A}{2 \pi} e^{-\frac{i}{\hbar}\left(V t-\frac{m}{2} \frac{x^{2}}{t}\right)} \int_{s t p h} \frac{1}{\Omega-\Omega_{0}} e^{-\frac{1}{2}\left(1-\frac{\Omega_{r}}{\Omega_{s}}\right)^{2} \Omega_{s} t} d \Omega .
$$

It may be evaluated in Gauss approximation, 


$$
\psi_{s}(x, t)=\frac{i A}{2 \pi} \sqrt{\frac{-4 \pi i}{\Omega_{s} t}} \frac{\Omega_{s}}{\Omega_{s}-\Omega_{0}} e^{-\frac{i}{\hbar}\left(V t-\frac{m}{2} \frac{x^{2}}{t}\right)},
$$

if the width $\left(\phi_{s}^{\prime \prime}\right)^{-1 / 2}$ of the exponential phase factor at the saddle point is small compared to the distance over which the prefactor changes significantly,

$$
\phi_{s}^{\prime \prime}\left(\Omega_{s}-\Omega_{0}\right)^{2}=\frac{1}{2}\left(\frac{\Omega_{s}-\Omega_{0}}{\Omega_{s}}\right)^{2} \Omega_{s} t \gg 1 .
$$

The pole contribution (20) describes a monochromatic wave with the frequency $\omega=\omega_{0}$ of the source, with a front which travels with velocity $v_{m}$ given by Eq. (19). In the propagating case $\hbar \Omega_{0}>0, v_{m}$ is equal to the group velocity $v_{g}=d \omega / d k$; in the evanescent case $\hbar \Omega_{0}<0$, $v_{m}$ is related to the traversal time for tunnelling [1] $\tau$ for large barrier widths $L$ by $v_{m}=L / \tau$.

The saddle contribution (21), on the other hand, describes the perturbation due to the switching-on of the source at $t=0$ which contains arbitrarily high frequencies; its front travels with the maximum speed permitted by the wave equation, which is infinite for a non-relativistic particle.

Pole contribution and saddle contribution correspond to the "main part" and the "forerunner" of Sommerfeld and Brillouin, respectively [23,24. We will keep the expression "forerunner" for the saddle contribution, but will call the pole contribution the "monochromatic part" instead of "main part", because the latter expression is extremely misleading in the evanescent case where the monochromatic part is exponentially small compared to the forerunner.

It is important to point out that the existence of a wave front of the monochromatic part $\psi_{p}$ does not mean that the total wave function is discontinuous at $x=v_{m} t$. In fact, the solution of the Schrödinger equation is continuous for all $x>0, t>0$. Thus, the pole contribution $\psi_{p}$ must combine at $x=v_{m} t$ with the saddle contribution $\psi_{s}$ in such a way that the total wave function $\psi$ is continuous.

This continuity requirement leads to an independent determination of the front velocity $v_{m}$ of the monochromatic part: The condition that the real parts of the phases of $\psi_{p}$ and $\psi_{s}$ coincide for $x=v_{m} t$ reads in the propagating case $\left(\Omega_{0}>0, k\left(\Omega_{0}\right)\right.$ real $)$

$$
\Omega_{0} t-k\left(\Omega_{0}\right) x=-\frac{m}{2 \hbar} \frac{x^{2}}{t} \quad \text { for } \quad x=v_{m} t
$$

and in the evanescent case $\left(\Omega_{0}<0, k\left(\Omega_{0}\right)\right.$ imaginary $)$

$$
-\left|\Omega_{0}\right| t=-\frac{m}{2 \hbar} \frac{x^{2}}{t} \quad \text { for } \quad x=v_{m} t
$$

which yields the same result as Eq. (19). This is important because one can raise an objection against the derivation based on the crossing of the stph-line with the pole: Although the stph-line is the natural choice for the saddle-point integration, this choice is by no means unique: the integral in Eq. (21) does not change if the path of integration is shifted away from the stph-line.

We now show explicitly that the crossing of the stph-line with the pole gives rise to a discontinuity of the saddle contribution $\psi_{s}(x, t)$ at $x=v_{m} t$ which exactly compensates the discontinuity of $\psi_{p}(x, t)$ at the onset of the monochromatic wave. 
In the propagating case, in the integral of Eq. (21) we set $\Omega_{r}=\Omega_{s}(1+u)$ where $u$ is an integration variable which measures the distance from the saddle point $\Omega_{s}$ on the real $\Omega$-axis. ¿From Eq. (17), we obtain

$$
\Omega=\Omega_{s}\left(1+(1-i) u-\frac{i}{2} u^{2}\right) ; \quad d \Omega=\Omega_{s}(1-i-i u) d u .
$$

Then, the integral $I$ along the stph-line becomes

$$
I=\int_{-\infty}^{+\infty} \frac{1-i-i u}{(1-i) u-\frac{i}{2} u^{2}-u_{0}} e^{-\frac{1}{2} \alpha u^{2}} d u
$$

where we have introduced the abbreviations

$$
u_{0}=\frac{\Omega_{0}-\Omega_{s}}{\Omega_{s}}=\frac{\left(v_{m} t\right)^{2}-x^{2}}{x^{2}} ; \quad \alpha=\Omega_{s} t=\frac{x^{2}}{2 \Delta x v_{m} t}
$$

where $\Delta x=\hbar /\left(m v_{m}\right)$ equals (up to a factor of $2 \pi$ ) the de Broglie-wave length of a particle moving with velocity $v_{m}$. The parameter in the validity condition (23) for the Gauss approximation takes the form

$$
\left(\frac{\Omega_{s}-\Omega_{0}}{\Omega_{s}}\right)^{2} \Omega_{s} t=\alpha u_{0}^{2}=\left(\frac{v_{m}^{2} t^{2}-x^{2}}{x v_{m} t}\right)^{2} \frac{v_{m} t}{2 \Delta x}
$$

The Gauss approximation (22) remains valid as long as $\alpha u_{0}^{2} \gg 1$, which is satisfied except very close to $x=v_{m} t$. In the immediate neighborhood $x=v_{m} t$ where $\alpha u_{0}^{2} \ll 1$, on the other hand, the integral becomes

$$
I=\lim _{u_{1} \rightarrow \infty} \int_{-u_{1}}^{+u_{1}} \frac{1}{u-\frac{1}{2}(1+i) u_{0}} d u=i \pi \operatorname{sign} u_{0}
$$

which yields the saddle contribution

$$
\psi_{s}(x, t)=-\frac{1}{2} A e^{-\frac{i}{\hbar}\left(V t-\frac{m}{2} \frac{x^{2}}{t}\right)} \operatorname{sign}\left(v_{m} t-x\right) \quad\left(v_{m} t \rightarrow x\right) .
$$

Thus, in the propagating case, the saddle contribution has at $x=v_{m} t$ a jump of magnitude A.

In the evanescent case, the Gauss approximation (22) remains valid at $x=v_{m} t$ where $\Omega_{0}=-\Omega_{s}$. However, since the wave number $k\left(\Omega_{0}\right)$ is imaginary, the "main part" $\psi_{p}$ is exponentially small compared to the "forerunner" $\psi_{s}$, and must therefore be compared with the equally small contribution to the integral in Eq. (21) from a narrow region around the pole. We set $\Omega_{r}=\Omega_{s}(-1+u)$ where the integration variable $u$ measures the distance from the point $-\Omega_{s}$ on the real $\Omega$-axis, and $\Omega_{0}=-\Omega_{s}\left(1+w_{0}\right)$ such that

$$
w_{0}=\frac{\left|\Omega_{0}\right|-\Omega_{s}}{\Omega_{s}}=\frac{v_{m}^{2} t^{2}-x^{2}}{x^{2}} \quad \text { whereas } \quad u_{0}=\frac{\Omega_{0}-\Omega_{s}}{\Omega_{s}}=-\frac{v_{m}^{2} t^{2}+x^{2}}{x^{2}} .
$$

¿From Eq. (17), we obtain 


$$
\Omega=\Omega_{s}\left(-1+(1+i) u-\frac{i}{2} u^{2}\right) ; \quad d \Omega=\Omega_{s}(1+i-i u) d u
$$

The contribution to the integral from the narrow region $-u_{1}<u<u_{1}$, where $\left|w_{0}\right| \ll u_{1} \ll$ $1, \alpha u_{1} \ll 1$, is given by

$$
\Delta I=e^{-2 \alpha} \int_{-u_{1}}^{+u_{1}} \frac{1}{u+\frac{1}{2}(1-i) w_{0}} d u=i \pi e^{-2 \alpha} \operatorname{sign} w_{0}
$$

which yields the contribution to $\psi_{s}$

$$
\Delta \psi_{s}(x, t)=-\frac{1}{2} A e^{-\frac{i}{\hbar}\left(V t-\frac{m}{2} \frac{x^{2}}{t}\right)} e^{-\frac{m}{\hbar} \frac{x^{2}}{t}} \operatorname{sign}\left(v_{m} t-x\right) \quad\left(v_{m} t \rightarrow x\right)
$$

Thus, in the evanescent case, the jump of the saddle contribution at $x=v_{m} t$ is exponentially small.

Comparison with Eq. (20) shows that $\psi_{s}$ has indeed in both cases a discontinuity opposite to that of $\psi_{p}$.

To summarize: In the propagating case the wave as observed at a distance $x$ from the source grows to one half of the asymptotic amplitude $\mathrm{A}$ at a time $t=x / v_{m}$ when the monochromatic (main) contribution of the source sets in. In contrast, in the evanescent case the sharp onset of the source generates a contribution to the wave which largely determines what is seen at the observation point. The saddle point solution is of order $A$ at $t=x / v_{m}$ when the exponentially small monochromatic contribution arrives. For a Schrödinger-like dispersion there is no limiting velocity, the sharp onset of the wave generates immediately a wave at the observation point $x$. Next we investigate a medium with dispersion which contains a limiting velocity.

\section{Relativistic particle}

The phase function $\phi(\Omega)$ in the complex $\Omega$-plane is shown in Figs. 2 and 3 for $x>c t$ and $x<c t$, respectively. It has branch cuts $\hbar \Omega=\left(-\infty \ldots-m c^{2}\right)$ and $\hbar \Omega=\left(+m c^{2} \ldots+\infty\right)$, and the values of $\phi(\Omega)$ on the upper and lower sheet are again related by Eq. (14).

For $x>c t, \operatorname{Im} \phi(\Omega) \rightarrow-\infty$ for $\operatorname{Im} \Omega \rightarrow+\infty$ (see Fig. 2). By shifting the path of integration upwards, one recognizes that

$$
\psi(x, t)=0 \text { for all }(x, t) \text { with } \quad x>c t
$$

as required by relativistic causality.

For $x<c t$, on the other hand, $\operatorname{Im} \phi(\Omega) \rightarrow-\infty$ only for $\operatorname{Im} \Omega \rightarrow-\infty$ (see Fig. 3). In this case, the phase factor $e^{-i \phi(\Omega)}$ has saddle points $\Omega= \pm \Omega_{s}$ on the real axis. For the saddle at $+\Omega_{s}$, the saddle-point condition $d \phi /\left.d \Omega\right|_{s}=0$ yields

$$
\hbar \Omega_{s}=m c^{2} \frac{t}{\vartheta}, \quad \hbar k_{s}=m \frac{x}{\vartheta}, \quad \hbar \phi_{s}=\hbar \Omega_{s} \frac{\vartheta^{2}}{t}=m c^{2} \vartheta
$$

where we have introduced the quantity 


$$
\vartheta(x, t)=\sqrt{t^{2}-\frac{x^{2}}{c^{2}}}
$$

The second derivative of the phase function at the saddle $+\Omega_{s}$ is

$$
\phi_{s}^{\prime \prime}=\left.\frac{d^{2} \phi}{d \Omega^{2}}\right|_{s}=\frac{\hbar}{m} \frac{\vartheta^{3}}{x^{2}}
$$

The line $s t p h^{+}$of stationary phase $\operatorname{Re} \phi(\Omega)=\phi_{s}$ through the saddle $+\Omega_{s}$, on which $\operatorname{Im} \phi$ decreases to $-\infty$ as one moves away from the saddle point, is given by

$$
\Omega_{i}=-\frac{\left(\Omega_{r}-\Omega_{s}\right)\left[\hbar^{2} \Omega_{r} \Omega_{s}-\left(m c^{2}\right)^{2}\right]}{\sqrt{\left[\hbar^{2} \Omega_{s}^{2}-\left(m c^{2}\right)^{2}\right]\left[-\hbar^{2} \Omega_{r}^{2}+2 \hbar^{2} \Omega_{r} \Omega_{s}-\left(m c^{2}\right)^{2}\right]}} .
$$

It crosses the real axis at the saddle point $\Omega=\Omega_{s}$ and at $\Omega=\left(m c^{2} / \hbar\right)^{2} / \Omega_{s}$, and goes to $\Omega_{i}=-\infty$ for $\Omega_{r}=\Omega_{s} \pm \sqrt{\Omega_{s}^{2}-\left(m c^{2} / \hbar\right)^{2}}$. In contrast to the non-relativistic case, it is no longer possible to obtain the imaginary part of the phase function $\phi(\Omega)$ on the stph-lines in closed analytic form. At the crossing point $\Omega=\left(m c^{2} / \hbar\right)^{2} / \Omega_{s}$, its imaginary part has the value $\operatorname{Im} \phi=-i m x^{2} / \hbar t$, which is the same as the value of $\operatorname{Im} \phi$ in the nonrelativistic case at the crossing point $\Omega=-\Omega_{s}$.

The results for the saddle at $-\Omega_{s}$ are obtained from Eqs. (37-40) by substituting $\Omega_{r} \mapsto$ $-\Omega_{r}, k_{r} \mapsto-k_{r}, \phi_{r} \mapsto-\phi_{r}$.

For a fixed point $x$ in space, the crossing points of the $s t p h^{+}$-line with the real axis move towards $\Omega=m c^{2}$ as $t$ increases form $x / c$ to $\infty$. In the propagating case, the saddle point $\Omega_{s}$ crosses the pole $\Omega=\Omega_{0}>m c^{2} / \hbar$ at time $t=x / v_{m}$ where $v_{m}$ is given by

$$
v_{m}=c \sqrt{1-\left(\frac{m c^{2}}{\hbar \Omega_{0}}\right)^{2}} \quad\left(\hbar \Omega_{0}>m c^{2}\right)
$$

which agrees with the group velocity $v_{g}=d \omega / d k$. In the evanescent case, the point $\Omega=$ $\left(m c^{2} / \hbar\right)^{2} /\left(\Omega_{s}\right)$ crosses the pole $\Omega=\Omega_{0}<m c^{2} / \hbar$ at time $t=x / v_{m}$ where $v_{m}$ is given by

$$
v_{m}=c \sqrt{1-\left(\frac{\hbar \Omega_{0}}{m c^{2}}\right)^{2}} \quad\left(\hbar \Omega_{0}<m c^{2}\right),
$$

which is equal to $\hbar|k| / m$. We relate $v_{m}$ to the relativistic traversal time for tunneling $\tau_{t r}$ for large barrier widths $L$ by $v_{m}=L / \tau$.

The velocity $v_{m}$ exceeds the velocity of light $c$ neither in the propagating nor in the evanescent case; it becomes equal to $c$ only for zero energy and in the limit of infinite energy. The energy dependence of $v_{m}$ is shown in Fig. 4 .

In order to identify the contributions to the wave functions $\psi(x, t)$, we proceed in the same way as in the non-relativistic case. The path of integration is deformed away from the real $\Omega$-axis such that it coincides with the $s t p h^{ \pm}$-lines at positive and negative energies. As long as $t<x / v_{m}$, no obstacle is encountered, and the integral remains unchanged; but for $t>x / v_{m}$ there occurs a loop around the pole which gives rise to a pole contribution 


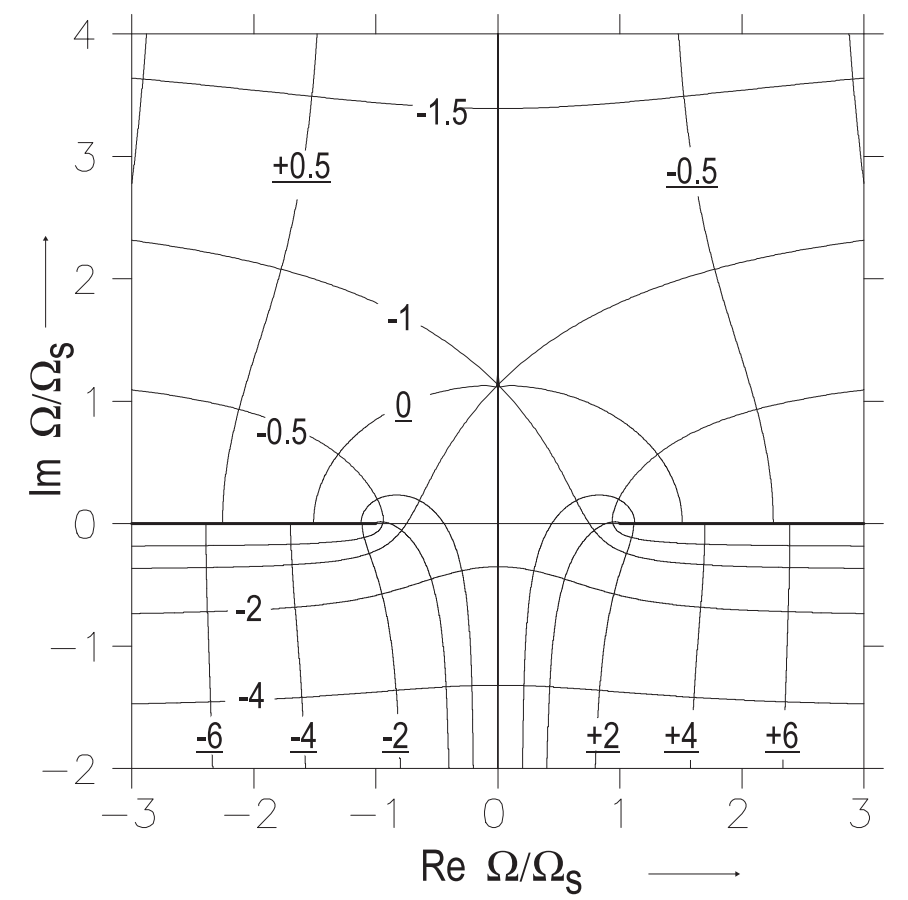

FIG. 2. Form of the phase function $\phi(\Omega)$ in the complex $\Omega$-plane for a relativistic particle for $c t=0.75 x$. Shown are the lines of constant $\varphi_{r}=\hbar \operatorname{Re} \phi /\left(m c \sqrt{x^{2}-c^{2} t^{2}}\right)$ for $\varphi_{r}=-6 \ldots+6\left(\Omega_{i}<0\right),=0, \pm 0.5\left(\Omega_{i}>0\right)$ (underlined numbers) and $\varphi_{i}=\hbar \operatorname{Im} \phi /\left(m c \sqrt{x^{2}-c^{2} t^{2}}\right)$ for $\varphi_{i}=-4,-2,-1.5,-1,-0.5$. After Ref. 31.

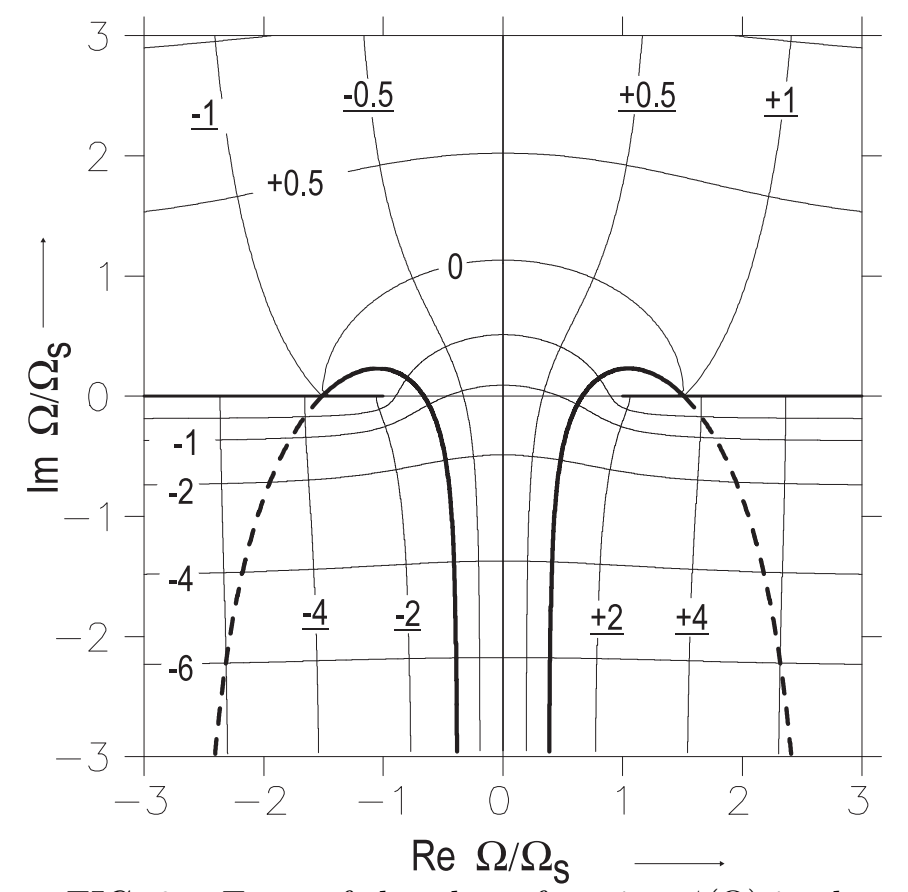

FIG. 3. Form of the phase function $\phi(\Omega)$ in the complex $\Omega$-plane for a relativistic particle for $c t=1.25 x$. Shown are the lines of constant $\varphi_{r}=\hbar \operatorname{Re} \phi /\left(m c \sqrt{c^{2} t^{2}-x^{2}}\right)$ for $\varphi_{r}=-6 \ldots+6$, and $\varphi_{i}=\hbar \operatorname{Im} \phi /\left(m c \sqrt{c^{2} t^{2}-x^{2}}\right)$ for $\varphi_{i}=-6 \ldots+1$. The branch cuts are indicated by an increased line thickness. The parts of the lines of stationary phase $\operatorname{Re} \phi=\phi_{s}^{ \pm}$on the upper and lower sheet are shown as full and dashed thick lines, respectively. After Ref. [31]. 


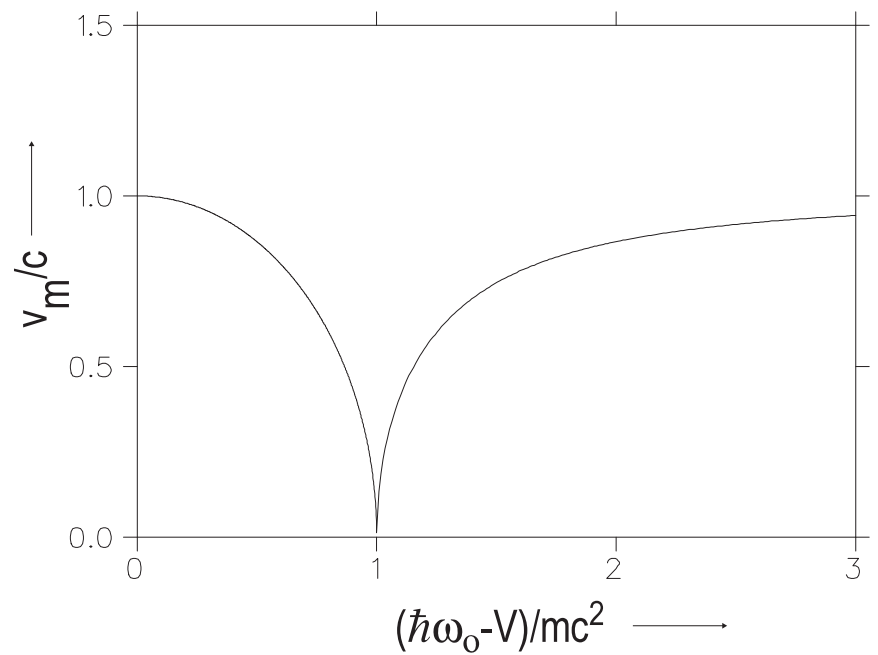

FIG. 4. Front velocity of the monochromatic wave for a relativistic particle as function of energy. After Ref. [31].

$$
\psi_{p}(x, t)=A e^{-i\left(\frac{1}{\hbar} V+\Omega_{0}\right) t} e^{i k\left(\Omega_{0}\right) x} \Theta\left(v_{m} t-x\right),
$$

describing a monochromatic wave with front velocity $v_{m}$ given by Eqs. (41,42). The integral along the $s t p h^{ \pm}$-lines yield saddle contributions

$$
\psi_{s}^{ \pm}(x, t)=\frac{i A}{2 \pi} e^{-i\left(\frac{1}{\hbar} V t \pm \phi_{s}\right)} \int_{s t p h \pm} \frac{1}{\Omega-\Omega_{0}} e^{-\operatorname{Im} \phi_{i}(\Omega)} d \Omega \Theta(c t-x) .
$$

with a front which moves with velocity $c$. The contribution due to the saddle at $+\Omega_{s}$ describes the excitation of particles, and that due to the saddle at $-\Omega_{s}$ the excitation of antiparticles. The latter part will be significant only for energies deep in the classically forbidden region, $\hbar \Omega_{0} \ll m c^{2}$. The saddle contributions may be evaluated in Gauss approximation,

$$
\psi_{s}^{ \pm}(x, t)=\frac{i A}{2 \pi} \sqrt{\frac{\mp 2 \pi i \hbar}{m c^{2} \vartheta} \frac{x^{2}}{c^{2} t^{2}}} \frac{\Omega_{s}}{\Omega_{s} \pm \Omega_{0}} e^{-\frac{i}{\hbar}\left(V t \pm m c \sqrt{c^{2} t^{2}-x^{2}}\right)} \Theta(c t-x) .
$$

if

$$
\phi_{s}^{\prime \prime}\left(\Omega_{s} \mp \Omega_{0}\right)^{2}=\frac{\hbar}{m} \frac{\vartheta^{3}}{x^{2}}\left(\Omega_{s} \mp \Omega_{0}\right)^{2} \gg 1 .
$$

Since the solution of the wave equation is continuous for all $x<c t$, the pole contribution $\psi_{p}$ must combine at $x=v_{m} t$ with the saddle contribution $\psi_{s}$ in such a way that the total wave function $\psi$ is continuous. This requirement leads again to an independent determination of the front velocity $v_{m}$ of the monochromatic part: The condition that the real parts of the phases of $\psi_{p}$ and $\psi_{s}$ coincide for $x=v_{m} t$ reads in the propagating case $\left(\hbar \Omega_{0}>m c^{2}, k\left(\Omega_{0}\right)\right.$ real)

$$
\hbar\left(\Omega_{0} t-k\left(\Omega_{0}\right) x\right)=m c \sqrt{c^{2} t^{2}-x^{2}} \text { for } \quad x=v_{m} t
$$

and in the evanescent case $\left(\hbar \Omega_{0}<m c^{2}, k\left(\Omega_{0}\right)\right.$ imaginary $)$ 


$$
\hbar \Omega_{0} t=m c \sqrt{c^{2} t^{2}-x^{2}} \text { for } \quad x=v_{m} t
$$

which yields the same result as Eqs. (41,42).

Like for a non-relativistic particle, it can be shown explicitly that the crossing of the $s t p h^{+}$-line with the pole gives rise to a discontinuity of the saddle contribution $\psi_{s}^{+}(x, t)$ at $x=v_{m} t$ which exactly compensates the discontinuity of $\psi_{p}(x, t)$ at the onset of the monochromatic wave. One obtains in the propagating case

$$
\psi_{s}^{+}(x, t)=-\frac{1}{2} A e^{-\frac{i}{\hbar}\left(V t+m c^{2} \vartheta\right)} \operatorname{sign}\left(v_{m} t-x\right) \quad\left(v_{m} t \rightarrow x\right) .
$$

and in the evanescent case

$$
\Delta \psi_{s}^{+}(x, t)=-\frac{1}{2} A e^{-\frac{i}{\hbar}\left(V t+m c^{2} \vartheta\right)} e^{-\frac{m}{\hbar} \frac{x^{2}}{t}} \operatorname{sign}\left(v_{m} t-x\right) \quad\left(v_{m} t \rightarrow x\right) .
$$

Like for the non-relativistic particle, in the evanescent case the saddle point contribution far exceeds the monochromatic contributions to the total wave.

\section{Fronts of a frequency-band limited source}

Clearly, the high frequencies generated by a source which is switched on instantly are highly undesirable. In this section we investigate a source which is limited in the frequency band width. The fact that experimental signals are often frequency-band limited has already been emphasized [32,33] (that is of course a technical and not a fundamental limitation [34]). We restrict the discussion to the case of a non-relativistic particle. The frequencyband limited source is described by an amplitude in Fourier space which is the product $\hat{A}\left(\omega-\omega_{0}\right) \hat{\chi}(\omega)$ of the amplitude $\hat{A}=i A /\left(\omega-\omega_{0}+i 0^{+}\right)$of the source with a mathematically sharp onset and a characteristic function $\hat{\chi}(\omega)$ which limits the range of frequencies.

For simplicity, we take

$$
\hat{\chi}(\omega)=\Theta\left(\omega-\left(\omega_{0}-\Delta \omega\right)\right)-\Theta\left(\omega-\left(\omega_{0}+\Delta \omega\right)\right),
$$

where $\Theta$ is the step function, although strictly speaking this form violates causality: As can be seen from the Fourier transform,

$$
\chi(t)=\frac{1}{\pi} e^{-i \omega_{0} t} \frac{\sin (\Delta \omega t)}{t},
$$

the source now radiates already for $t<0$. The frequency band width $\Delta \omega$ is chosen such that

$$
\frac{1}{\tau} \ll \Delta \omega \ll \frac{V}{\hbar}-\omega_{0}=\left|\Omega_{0}\right|
$$

Here the lower limit ensures that the onset of the source is still fast compared to the traversal time $\tau=x / v_{m}$ and that the effect of causality violation is kept mall. The upper limit assures that all frequencies contained in the source are in the evanescent region. 
We start with the integral for the wave function given by Eq. (12). The integration path is originally along the real axis from $\Omega_{-}=\Omega_{0}-\Delta \omega$ to $\Omega_{+}=\Omega_{0}+\Delta \omega$ above the pole at $\Omega_{0}$. It is now convenient to deform the integration path in such a way that the path first follows the line of constant imaginary phase $\operatorname{Im} \phi\left(\Omega_{-}\right)=\phi_{i}^{-}$which goes through the point $\Omega_{-}$, then from the point where this line (see Fig. 1) intersects the stph-line follows the line stph to the point where it is intersected by the line of constant imaginary phase $\operatorname{Im} \phi\left(\Omega_{+}\right)=\phi_{i}^{+}$, and then follows this line to the point $\Omega_{+}$. For $\Omega_{0}<-\Omega_{s}$, we have to pull the integration path across the pole, which yields a monochromatic contribution to the wave function

$$
\psi_{p}(x, t)=A e^{-i\left(\frac{1}{\hbar} V+\Omega_{0}\right) t} e^{i k\left(\Omega_{0}\right) x} \Theta\left(v_{m} t-x\right),
$$

of the same form as Eq. (20).

The other three contributions to the integral are evaluated for a time interval for which $-\Omega_{s}$ is close to the pole $\Omega_{0}$. Furthermore, we are interested in sources with a band width which is small compared to $\left|\Omega_{0}\right|$. To investigate this time window, we can therefore linearize the phase function $\phi$ around the frequency $-\Omega_{s}$. In terms of the variables $u$ and $v$ which measure the deviation from $\Omega_{s}$, i.e. $\Omega=\Omega_{s}(-1+u+i v)$, we find for the phase function $\phi_{r}=\operatorname{Re} \phi=\Omega_{s} t(-1+u-v)$ and $\phi_{i}=\operatorname{Im} \phi=\Omega_{s} t(-2+u+v)$. Locally, around $\Omega=-\Omega_{s}$ the lines of stationary phase form thus a rectangular grid. In terms of $u$ the upper and lower frequency limits are

$$
u_{ \pm}=\frac{\Omega_{s}-\left|\Omega_{0}\right|}{\Omega_{s}} \pm \frac{\Delta \omega}{\Omega_{s}}=-w_{0} \pm \frac{\Delta \omega}{\Omega_{s}} .
$$

We recall from Eq (32) that $w_{0}=\left(v_{m}^{2} t^{2}-x^{2}\right) / x^{2}$. The first part $I_{1}$ of the integral along the line $\phi_{i}^{-}=\Omega_{s} t\left(-2+u_{-}\right)$extends in $u$ from $u_{-}$to $u_{-} / 2$, the second part $I_{2}$ of the integral along the stph-line with $\phi_{r}=-\Omega_{s}$ t extends in $u$ from $u_{-} / 2$ to $u_{+} / 2$, and the third integral $I_{3}$ along $\phi_{i}^{+}=\Omega_{s} t\left(-2+u_{+}\right)$extends in $u$ from $u_{+} / 2$ to $u_{+}$.

The first (index - ) and the third integral (index + ) is approximated by pulling the denominator for $u=u_{ \pm}$in front,

$$
\psi_{ \pm}(x, t) \approx \frac{(1+i) A}{2 \pi} \frac{1}{\Delta \omega t} e^{-\frac{i}{\hbar}\left(V t-\frac{m}{2} \frac{x^{2}}{t}\right)} e^{-\frac{m}{\hbar} \frac{x^{2}}{t}} e^{\left(-\frac{i}{2}+1\right) \Omega_{s} t u_{ \pm}} \sin \left(\Omega_{s} t \frac{u_{ \pm}}{2}\right)
$$

For $\left|w_{0}\right| \gg \Delta \omega /\left|\Omega_{0}\right|$, which holds outside a narrow time interval around $t=\tau$, the second integral is approximated by pulling the denominator at $u=-w_{0}$ in front,

$$
\psi_{2}(x, t) \approx \frac{(1+i) A}{2 \pi} \frac{1}{\left(\Omega_{s}-\left|\Omega_{0}\right|\right) t} e^{-\frac{i}{\hbar}\left(V t-\frac{m}{2} \frac{x^{2}}{t}\right)} e^{-\frac{m}{\hbar} \frac{x^{2}}{t}} e^{\left(\Omega_{s}-\left|\Omega_{0}\right|\right) t} \sinh (\Delta \omega t) .
$$

The key point is that all three integrals are exponentially suppressed. For $t=\tau$ we have $\exp \left(-m x^{2} /(2 \hbar \tau)\right)=\exp \left(-m v_{m} x / \hbar\right)$. The second point to notice is that because we still have a range of frequencies, the uppermost frequencies are least suppressed. Thus at $t=\tau$ the upper frequencies are enhanced by a factor $\exp \left(\Omega_{s} \tau u_{+}\right)=\exp \left(\left(m v_{m} x / \hbar\right) \Delta \omega /\left|\Omega_{0}\right|\right)$ whereas the frequencies at the lower end of the spectrum are additionally suppressed by a factor $\exp \left(\Omega_{s} \tau u_{-}\right)=\exp \left(-\left(m v_{m} x / \hbar\right) \Delta \omega /\left|\Omega_{0}\right|\right)$. Clearly the different exponential suppression of these frequencies is unavoidable. 
Let us next discuss what happens in the narrow time interval around $t=\tau$ where $\left|w_{0}\right| \ll \Delta \omega /\left|\Omega_{0}\right|$, and where the expression for the wave function $\psi_{2}(x, t)$ obtained above is not valid. For this integral, we have to reconsider a discussion analogous to that which leads to Eq. (34). For $w_{0} \neq 0$, by pulling the exponential factor at the upper limit in front, we find

$$
\psi_{s, 2}(x, t) \approx \frac{A}{\pi} e^{-\frac{i}{\hbar}\left(V t-\frac{m}{2} \frac{x^{2}}{t}\right)} e^{-\frac{m}{\hbar} \frac{x^{2}}{t}} e^{\Delta \omega t} \arctan \left(\frac{\Delta \omega}{\Omega_{s}-\left|\Omega_{0}\right|}\right) .
$$

At $w_{0}=0$, on the other hand, the integral has a discontinuity which exactly compensates the jump of the pole contribution $\psi_{p}$.

The discussion presented here does not show explicitly the short-time and the longtime behavior of the stationary phase solution. This restriction is due to the linearization of the phase function. If we are sufficiently far away from the point $\Omega_{0}=-\Omega_{s}$, we can estimate the contribution from the saddle-point solution near the intersection point of the stph-line with the vertical line that runs through $\Omega_{+}$. This leads to a wave function whose magnitude is governed by $\exp \left(-\frac{1}{2} \Omega_{s} t\left(1-\left(\Omega_{+} / \Omega_{s}\right)^{2}\right)\right)$. For times which are short compared to $\tau$, the saddle-point frequency $\Omega_{s}$ is large compared to $\left|\Omega_{+}\right|$, and the exponential can be approximated by $\exp \left(-\frac{1}{2} \Omega_{s} t\right)$. For times which are large compared to $\tau$, we have $\Omega_{s} \ll\left|\Omega_{+}\right|$, and the exponential can be approximated by $\exp \left(-\frac{1}{2} \Omega_{s} t\left(\Omega_{+} / \Omega_{s}\right)^{2}\right)$ which decays with time as $\exp \left(-\right.$ const. $\left.t^{3}\right)$. Thus the saddle point contribution is small both at times which are short compared to $\tau$ and at times which are long compared to $\tau$.

To summarize: By limiting the frequency band width of the source we obtain a wave evolution in the evanescent medium which shares the essential properties of the wave evolution in the propagating case: At a time $t=\tau$ a forerunner with a broad frequency distribution is augmented by a monochromatic front with the frequency of the source.

\section{Discussion}

In this work we have focused on the question of whether it is possible to find a traversal time which is associated with a causal process. The process investigated is the propagation of monochromatic fronts. For the case of a source with a sharp onset these monochromatic fronts are exponentially small compared to the forerunner which is not attenuated. However, as we have shown, if the source is frequency-band limited the forerunners are also exponentially attenuated. For the evanescent case, a wave evolution is found, which as in the propagating case, exhibits at $t=\tau$ a crossover from a wave with a broad frequency spectrum (forerunner) to a nearly monochromatic wave. We also emphasize that the total wave function, respectively, the resulting probability distribution is not the only observable. Possibly, an other way to make the monochromatic fronts visible is to incorporate a detector which is sensitive only to a narrow range of frequencies in an interval around the main frequency $\omega_{0}$ of the source. Or, we could consider particles with a spin in an evanescent medium with a weak magnetic field and could investigate the rotation of the spin as a function of time similarly to the stationary analysis of the Larmor clock [6]. Our work shows that the investigation of the time evolution of particle fields is a very intersting avenue of

research. The comparison of such investigations with the results from the oscillating barrier 
approach and quantum clocks [1, 6, 8] should be particularly illustrative. Furthermore, we hope that this work stimulates also experiments which aim to identify the monochromatic fronts discussed in this work.

\section{Acknowledgements}

M. B. has been a collaborator of Rolf Landauer for a number of years and has profited from his insights and stimulations much more than can be expressed in this brief and formal publication. H. T. has profited from a multitude of stimulating interactions with Rolf Landauer over a period of many years. We have benefited from discussions with P. Wittwer, and we thank T. Gyalog for help with the figures. 


\section{REFERENCES}

[1] M. Büttiker and R. Landauer, Phys. Rev. Lett. 49, 1739 (1982).

[2] M. Büttiker and R. Landauer, Physica Scripta 32, 429-434, (1985); Festkörperprobleme (Advances in Physics)XXV, edited by P. Grosse (Viehweg Braunschweig, 1985). p. 711; IBM J. Res. Dev. 30, 451 (1986).

[3] Th. Martin and R. Landauer, Phys. Rev. A 47, 2023 (1993).

[4] A. I. Baz', Sov. J. Nucl. Phys. 4, 182 (19967).

[5] V. F. Rybachenko, Sov. J. Nucl. Phys. 5, 635 (1967).

[6] M. Büttiker, Phys. Rev. B27, 6178 (1983).

[7] A. M. Steinberg, Phys. Rev. Lett. 74, 2405 (1995); V. Gasparian, M. Ortuno, J. Ruiz, and E. Cuevas, Phys. Rev. Lett. 75, 2312 (1995).

[8] R. Landauer and Th. Martin, Rev. of Modern Physics, 66, 217 (1994).

[9] J. E. Hauge and J. A. Stoveng, Rev. Mod. Phys. 61, 917 (1989).

[10] M. Büttiker, in "Electronic Properties of Multilayers and low Dimensional Semiconductors", edited by J. M. Chamberlain, L. Eaves, and J. C. Portal, (Plenum, New York, 1990). p. 297-315.

[11] C. R. Leavens and G. C. Aers, J. Vac. Sci. Technol. A 6, 305 (1988).

[12] J. M. Martinis, M. H. Devoret, D. Esteve, C. Urbina, Physica B152, 159 (1988); P. Gueret, E. Marclay and H. Meier, Appl. Phys. Lett. 53, 1617 (1988). Typically the time-scales in electric conductors reflect collective excitations: M. Büttiker, H. Thomas, and A. Pretre, Phys. Lett. A180, 364 - 369, (1993); T. Christen and M. Büttiker, Phys. Rev. Lett. 77, 143 (1996); P. W. Brouwer and M. Büttiker, Europhys. Lett. 37, 441-446 (1997).

[13] Th. Martin and R. Landauer, Phys. Rev. A45, 2611 (1992).

[14] A. M. Steinberg, P. G. Kwiat and R. Y. Chiao, Phys. Rev. Lett. 71, 708 (1993).

[15] A. Ranfagni, D. Mugnai, P. Fabeni, G. P. Pazzi, Appl. Phys. Lett. 58, 774 (1991). A. Ranfagni, P. Fabeni, G. P. Pazzi, and D. Mugnai, Phys. Rev. E48, 1453 (1993).

[16] G. Nimtz, A. Enders, and H. Speiker, J. Phys. I France 4, 565 (1994); A. M. Steinberg, J. Phys. I France 4, 1813 (1994); A. Enders, G. Nimtz, Phys. Rev. E 48, 632 (1993); Phys. Rev. B 47, 9605 (1993).

[17] Ch. Spielmann, P. Szipöcs, A. Stingl, and F. Krausz, Phys. Rev. Lett. 73, 2308 (1994).

[18] M. Deutsch and J. E. Golub, Phys. Rev. A 53, 434 (1996).

[19] P. Balcou and L. Dutriaux, Phys. Rev. Lett. 78, 851 (1997).

[20] J. M. Deutch and F. E. Low, Annals of Physics 288, 184 (1993).

[21] Y. Japha and G. Kurizki, Phys. Rev. A53, 586 (1996).

[22] A. Sommerfeld, Physikalische Zeitschrift 8, 841 (1908).

[23] A. Sommerfeld, Ann. Physik 44, 177 (1914).

[24] L. Brillouin, Ann. Physik 44, 203 (1914); Wave Propagation and Group Velocity, (Academic Press, New York, 1960).

[25] K. W. H. Stevens, Eur. J. Phys. 1, 98 (1980).

[26] N. Teranishi, A. M. Kriman, and D. K. Ferry, Supperlatt. Microstruct. 3, 509 (1987).

[27] A. P. Jauho and M. Jonson, Supperl. Microstruc. 6, 303 (1989).

[28] A. Ranfagni, D. Mugnai, A. Agresti, Phys. Lett. A158, 161 (1991).

[29] P. Moretti, Physica Scripta, 48, 18 (1992); Phys. Rev. A 46, 1233 (1992). 
[30] S. Brouard and J. G. Muga, Phys Rev. A54, 3055 (1996).

[31] M. Büttiker and H. Thomas (unpublished).

[32] H. M. Brodowsky, W. Heitmann, G. Nimtz, Phys. Lett. A222, 125 (1996).

[33] A. Ranfagni and D. Mugnai, Phys. Rev. 52, 1128 (1995).

[34] M. Mitchell and R. Chiao, Phys. Lett. A230, 133 (1997). 